\title{
An Equal Area Quad-tree Global Discrete Grid Subdivision Algorithm Based on Latitude and Longitude Lines
}

\author{
Wu Huixin ${ }^{*}$ and Li He \\ Department of Information Engineering, North China University of Water Resources and Electric Power, Zhengzhou, \\ 450046, China
}

\begin{abstract}
In order to effectively solve the problems of global discrete grid in spatial data management and operations, a novel equal area quad-tree global discrete grid subdivision model based on latitude and longitude lines is proposed in this paper. Coding rules of grid cells, and geometric distortion calculation and analysis of the grid model are also given in the detailed subdivision process. The results have shown that the proposed grid cell has simple structure and stable geometric distortion. Meanwhile, it features hierarchy and nested structure and thus facilitates multi-resolution modelling.
\end{abstract}

Keywords: Global Discrete Grid, Quad-tree Grid, Geometric Distortion, Equal Area, GIS.

\section{INTRODUCTION}

With the deepening research of global climate, global resource management and environmental protection, and the study of global issues such as global wildlife and ecological monitoring, more and more applications need to operate on a global scale. And a series of problems will appear if spherical or ellipsoidal surface data is converted to planar data with the way of map projection for processing: the computing of length and area will have significant deviations, and data overlap, fracture and inconsistencies of spatial relationships will occur. Seamless links of worldwide spatial data cannot be achieved, and the validity and accuracy of the calculation are questioned.

In order to break through the plane restrictions and store, manage and express spatial information according to the real shape of the Earth, the global discrete grid subdivision method come into being. The global discrete grid is a spherical surface-based fitting grid which can be infinitely subdivided, but does not intend to change the spherical shape. When it is subdivided into a certain extent, we can achieve the purpose of simulating the earth's surface. The grid, which features hierarchy and global continuity, not only avoiding the distortion of angle, length and area and discontinuity of spatial data brought by planar projection, but also overcoming many constraints and uncertainty which limits the GIS application, that is, the spatial data with any resolution (different precision) captured at any location on the planet can be expressed and analyzed normatively, and multi-resolution operations with specified precision can also be carried out on it. The method not only can solve the data fracture, distortion and topology inconsistency of planar models in global multi-scale spatial data management, but also can easily integrate, share and utilize spatial information resources in the grid computing environment [1]. How to divide the earth's surface into an equal-area and equal-shape hierarchy structure with multiple resolutions -- that is, the shape of each cell after each subdivision remains unchanged and all cells of each level are congruent (equal side length and angle) -- is the main content of researches on spherical discrete grid subdivision methods. However, we know from the spherical geometry that, unlike the planar grid cells, no method can allow spherical grid cells at each level to have identical geometric characteristics (such as size, length and angle), their results can only be approximately equal.

\section{REVIEW OF THE STATUS OF CURRENT RESEARCHES}

At present, the global discrete grid subdivision methods can be divided into three main categories: global discrete grid subdivision method based on latitude and longitude lines, global discrete grid subdivision method based on regular polyhedron and adaptive global discrete grid subdivision method.

\subsection{Global Discrete Grid Subdivision Method Based on Latitude and Longitude Lines}

The global discrete grid subdivision method based on latitude and longitude lines, which is the earliest and most widely used geospatial grid in geoscience, utilizes latitude and longitude lines with certain intervals (constant interval or variable interval) to split the global spherical surface. It accords with people's habits of mind. Its data structure is simple and thus it is easy to convert to other coordinate systems (vice versa). This method is also the basis of many existing datasets, algorithms and software, thus facilitating the use of existing data. Global discrete grid based on 
latitude and longitude lines can be divided into constantinterval and variable-interval global discrete grid [5]. Constant-interval global discrete grid based on latitude and longitude lines has obvious deficiencies: with the increase of latitude, changes of area and shape of the grid cells are getting bigger, and distances between the grid points are getting shorter, resulting in a significant data redundancy which is not conducive to the operation of global multiresolution data [6-8].

In order to overcome the deficiencies of the constantinterval subdivision, some institutions and scholars adopt a more flexible method -- variable-interval global discrete grid based on latitude and longitude lines. For example, the Digital Terrain Elevation Data (DTED) provided by the American National Imagery and Mapping Agency (NIMA) (Fig. 1) and FFI grid proposed by Bjørke et al. (Fig. 2).

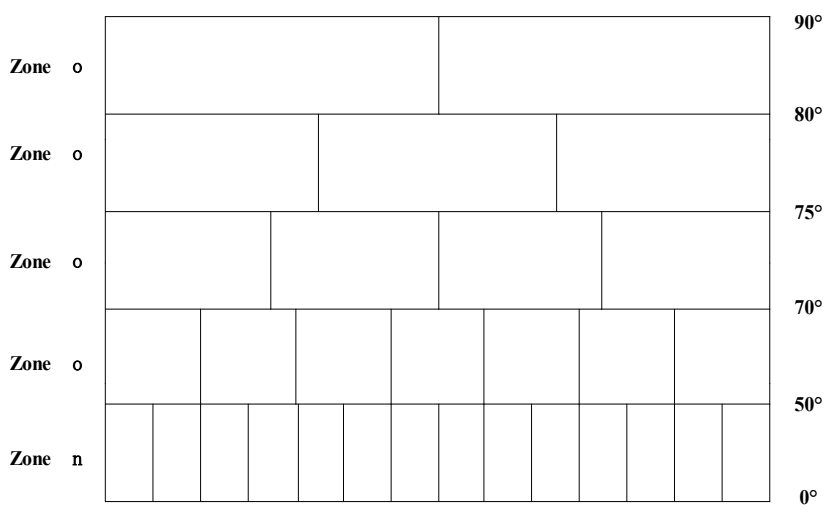

Fig. (1). DTED data grid structure (NIMA 2003).

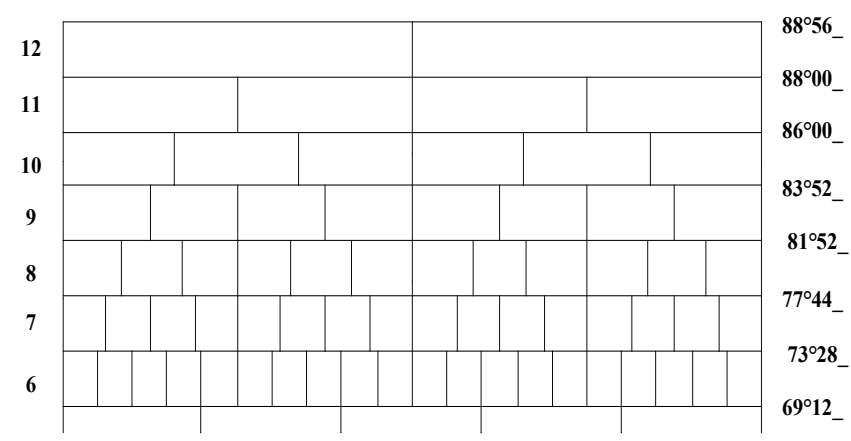

Fig. (2). FFI grid structure (Bjørke et al., 2003).

Compared with the constant-interval global the discrete grid based on latitude and longitude lines, DTED grid has lessened the data redundancy to some extent, but its grid division is still asymmetrical. Bjørke's FFI grid ensures that the grid area is roughly equal and the grid is more even. It lessened data redundancy and is easy to complete surface modeming based on discrete points, and can facilitate statistical calculations. However, it does not have hierarchy and nested structure, so continuous multi-resolution surface modelling is difficult to be carried out with this method.

\subsection{Global Discrete Grid Subdivision Method Based on Regular Polyhedron}

The global discrete grid subdivision method based on regular polyhedron utilizes hierarchical recursive subdivision on the basis of sphere-inscribed regular polyhedrons to form a grid model which covers the global spherical surface. There are five kinds of ideal inscribed regular polyhedrons in a sphere: regular tetrahedron (4 equilateral triangles), regular hexahedron (6 squares), regular octahedron (8 triangles), regular dodecahedron (12 pentagons) and regular icosahedron (20 equilateral triangles). These polyhedrons can produce identical spherical polygons when they are projected onto a spherical surface, and the number of polygons associated with each vertex is equal. So it is suitable for them to act as the basis of spherical subdivision (Fig. 3). The global discrete grid subdivision methods based on regular polyhedron usually use triangle, diamond and hexagon structures, and its biggest drawback is that it is difficult to converge and be compatible with data of the existing quadrilateral structures, therefore, corresponding geographic coordinate conversion must be carried out on it to utilize the existing data.
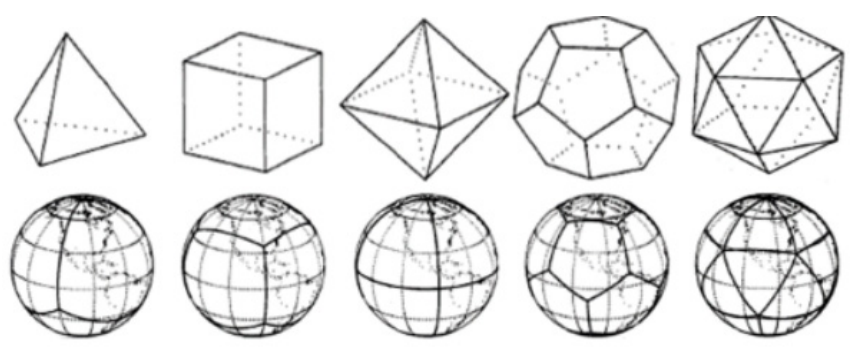

Fig. (3). The five ideal polyhedrons and their projections on the spherical surface.

\subsection{Adaptive Global Discrete Grid Subdivision Method}

Based on the entity elements on a spherical surface, the adaptive global discrete grid subdivision method divides the surface by using some kind of characteristics of the entities. Lukatela, Kolar and Most-afavi et al. proposed a variety of Voronoi-based adaptive discrete spherical grid subdivision methods in which grid cells can be adaptively adjusted according to the density of the entity data. Voronoi-based adaptive grid is more flexible than the grid generated by regular or semi-regular subdivision methods. However, the recursive subdivision of Voronoi grid is very difficult and it is almost impossible to establish a multi-resolution pyramid structure, which is very negative for applications that need multi-scale data support, such as three-dimensional visualization of large-scale scenarios. Application cases of two types of adaptive grids are shown in Fig. (4) [2].

\section{EQUAL AREA QUAD-TREE GLOBAL DISCRETE GRID SUBDIVISION ALGORITHM BASED ON LATITUDE AND LONGITUDE LINES}

The global discrete grid subdivision method based on latitude and longitude lines accords with people's habits of 


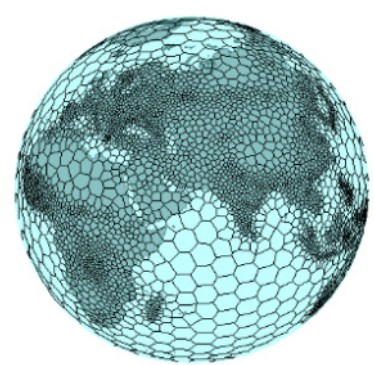

(a)

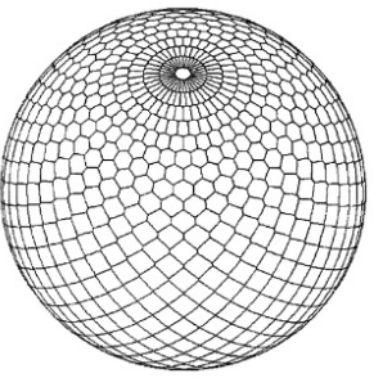

(b)
Fig. (4). Adaptive global discrete grid subdivision method: (a) Voronoi grid of the irregular entity; (b) Voronoi grid of the regular entity.

mind. Its data structure is simple and thus it is easy to be converted to other coordinate systems (vice versa). This method is also the basis of many existing datasets, algorithms and software, thus facilitating the use of existing data. Therefore, this method is the earliest and most widely used geospatial grid in geoscience. However, the nonuniformity of this kind of grid has restricted its applications, and therefore it is very necessary to improve the global discrete grid subdivision method based on latitude and longitude lines and figure out a subdivision method which not only has advantages of global discrete grid based on latitude and longitude lines but also overcomes its disadvantage of non-uniformity. For this end, an equal area global discrete grid subdivision method based on latitude and longitude lines is proposed in this paper. This algorithm can carry out equal-area, approximately-equal-shape, hierarchical multi-resolution grid subdivision on the earth's surface, providing a more effective global discrete grid subdivision method for the effective representation and management of global massive data.

\subsection{Principle of the Algorithm}

We take the sphere-inscribed regular octahedron as the basis of global discrete grid subdivision and carry out initial subdivision on the spherical surface: First of all, coincide the six vertices of the sphere-inscribed regular octahedron with the two poles of the sphere, the intersection point of the equator and the principal meridian, the intersection point of the equator and the $90^{\circ}$ meridian, the intersection point of the equator and the $180^{\circ}$ meridian and the intersection point of the equator and the $270^{\circ}$ meridian respectively; then, project the regular octahedron through the center of the sphere on the spherical surface to obtain 8 spherical triangles $R_{1}, R_{2}, \ldots, R_{8}$, completing the initial subdivision of the spherical surface (Fig. 5).

The first subdivision level: we use the arc of one weft to divide each spherical triangle $R_{\mathrm{i}}$ into 2 parts with equal area, obtaining 1 spherical subtriangle and 1 spherical trapezoid. Then we use the arcs of another warp and another weft to divide the spherical subtriangle and spherical trapezoid into 2 parts with equal area respectively, obtaining 1 spherical subtriangle $t_{i 1}$ and 3 spherical trapezoids $q_{i 1}, q_{i 2}$ and $q_{i 3}$, the first subdivision level is finished (Fig. 6), where $i=1,2$, ..., 8;
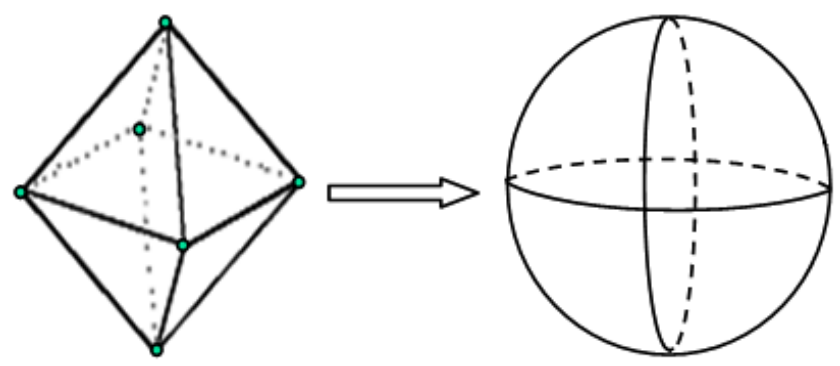

Fig. (5). Initial subdivision of the spherical surface using octahedron.

The second subdivision level: we first use the arcs of warp and weft to divide each spherical trapezoid $q_{i j}$ into 4 equal-area subtrapezoids $q_{i j 1}, q_{\mathrm{ij} 2}, q_{i j 3}$ and $q_{i j 4}$, where $i=1,2$, $\ldots, 8, j=1,2,3$; then we divide the spherical triangle $t_{i 1}$ into 4 equal-area parts according to the subdivision method of the first level -- the second subdivision level is finished, obtaining 16 equal-area parts totally[4];

Continue to carry out recursive subdivision like this until we obtain the required accuracy at the $n t h$ level, where $n$ is a natural number.

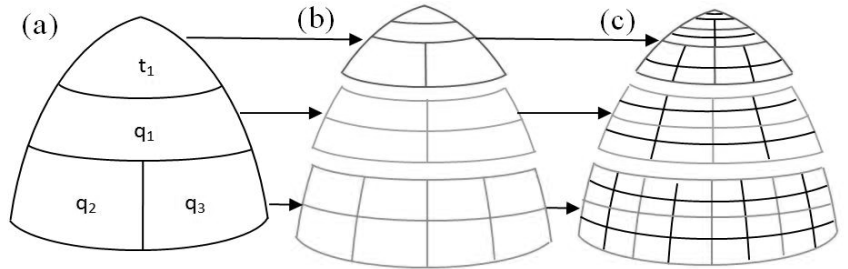

Fig. (6). Subdivision process of the equal area global discrete grid based on latitude and longitude lines.

The process can proceed infinitely following this method and each level of grid can form a quad-tree which is shown in Fig. 7. The root is the spherical triangle $R_{i}$ generated in the initial subdivision, followed by level 1 , level $2, \ldots$, level $n$ grid cells. The nth level has $4^{\text {n }}$ grid cells.

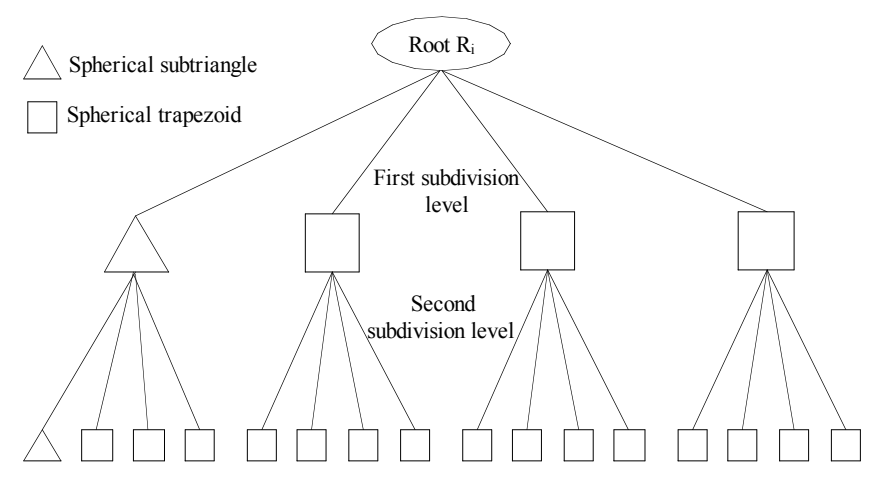

Fig. (7). Equal-area grid quad-tree. 


\subsection{Confirmation of Latitude and Longitude Lines of Each Level}

In order to complete the subdivision of the above principle, firstly we must determine the subdivision latitude and longitude lines of each grid level. The confirmation of the subdivision latitude and longitude lines of each grid level includes two cases, one is to determine the latitude and longitude lines of the spherical triangle grid, and the other is to determine the latitude and longitude lines of the spherical trapezoid grid.

\section{(1) Confirmation of latitude and longitude lines of the spherical triangle cell}

We use longitude lines $\lambda_{1}$ and $\lambda_{2}$ and latitude line $\varphi_{1}$ to denote the three sides of the spherical triangle respectively, and latitude lines $\varphi_{2}$ and $\varphi_{3}$ to represent the subdivision latitude lines and longitude line $\lambda_{3}$ to represent the subdivision longitude line (Fig. 8). And the longitude of the longitude line $\lambda_{3}$ is the mean value of the longitude of the two longitude lines of the spherical triangle, i.e. $\lambda_{3}=\frac{\lambda_{1}+\lambda_{2}}{2}$, while the latitude of latitude lines can be obtained by carrying out inverse solution based on the area of the triangle cell and the known latitude $\varphi_{1}$, the calculation is as the following:

\section{Known Conditions}

1) The radius of the sphere is $R$;

2) The area of the divided spherical triangle is $F . F$ can be calculated according to the subdivision levels due to equal-area subdivision, so it is known parameter, then:

$\frac{F}{2}=R^{2}\left(\lambda_{2}-\lambda_{1}\right)\left(\sin \varphi_{2}-\sin \varphi_{1}\right)$

So we can obtain: $\varphi_{2}=\arcsin \left[\frac{F}{2 R^{2}\left(\lambda_{2}-\lambda_{1}\right)}+\sin \varphi_{1}\right]$

Then we take $\varphi_{2}$ as a known parameter, similarly, we can obtain $\varphi_{3}: \varphi_{3}=\arcsin \left[\frac{F}{4 R^{2}\left(\lambda_{2}-\lambda_{1}\right)}+\sin \varphi_{2}\right]$

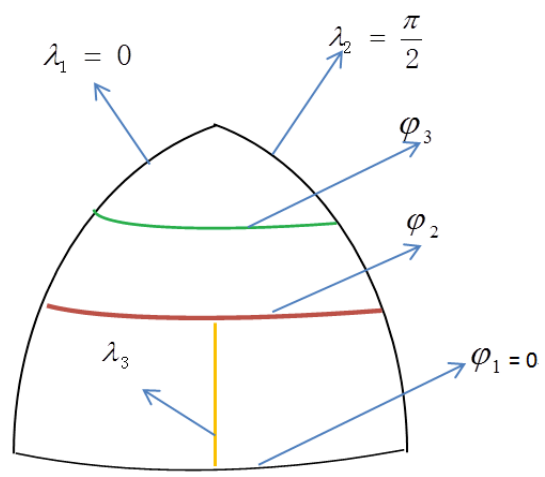

Fig. (8). Confirmation of diagram of latitude and longitude lines of the spherical triangle cell.
(2) Confirmation of latitude and longitude lines of the spherical trapezoid cell

We use longitude lines $\lambda_{1}$ and $\lambda_{2}$ and latitude lines $\varphi_{1}$ and $\varphi_{2}$ to denote the four sides of the spherical trapezoid respectively, latitude line $\varphi_{3}$ and longitude line $\lambda_{3}$ to represent the subdivision latitude and longitude lines (Fig. 9). And the longitude of the longitude line $\lambda_{3}$ is the mean value of the longitude of the two longitude lines of the spherical trapezoid, i.e. $\lambda_{3}=\frac{\lambda_{1}+\lambda_{2}}{2}$, while $\varphi_{3}$ can be obtained by carrying out inverse solution based on the area of the spherical trapezoid and the known latitude $\varphi_{1}$, and the calculation formula is as the following:

$\varphi_{3}=\arcsin \left[\frac{F}{2 R^{2}\left(\lambda_{2}-\lambda_{1}\right)}+\sin \varphi_{1}\right]$

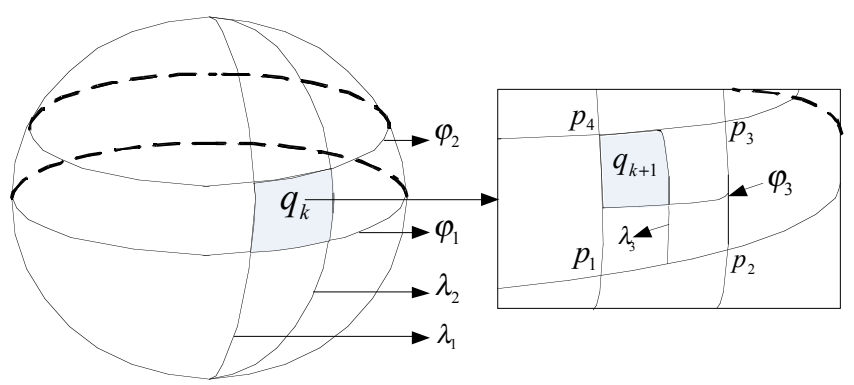

Fig. (9). Sketch diagram of latitude and longitude lines of the spherical trapezoid cell.

\section{ENCODING RULES OF GRID CELLS}

According to the above algorithm, the earth's surface can be divided into equal and adjacent grid cells based on certain sizes (resolution). Each grid cell, which is called a resolution pixel, has a unique attribute value and represents a small area of the earth's surface. The number of resolution pixels will increase exponentially with the increase of subdivision levels. Therefore, the resolution pixels need to be encoded to uniquely identify their spatial positions.

The octant obtained after the initial subdivision can be identified according to its location by one octant code ( 0 to 7), and the identification rules are shown in Fig. 10. Each octant can be expressed by a quad-tree, and the system just needs to record the location of its leaf node at storage. When the octant is recursively divided into smaller grid cells, the location of its leaf node can be identified with a quaternary Morton code.

Specific coding rules are as follows: for the initial octant (spherical triangle) in the first subdivision grid level, the top triangle is 0 , the middle spherical quadrilateral is 1 , and the two spherical quadrilaterals at bottom left and right corners are coded as 2 and 3; for the grid cells of the second level, the encoding method of the spherical triangle and initial 
subdivision octant cells is the same. For the spherical quadrilaterals, the top left and top right parts are coded as 1 and 0 respectively, and the bottom left and right parts are coded as 2 and 3 . The codes generated in this way have a fixed directionality and are conducive to the adjacent search and coordinate conversion of grid cells. The codes of other subdivision levels can be deduced by analogy..... The encoding process is shown in Fig. 11.

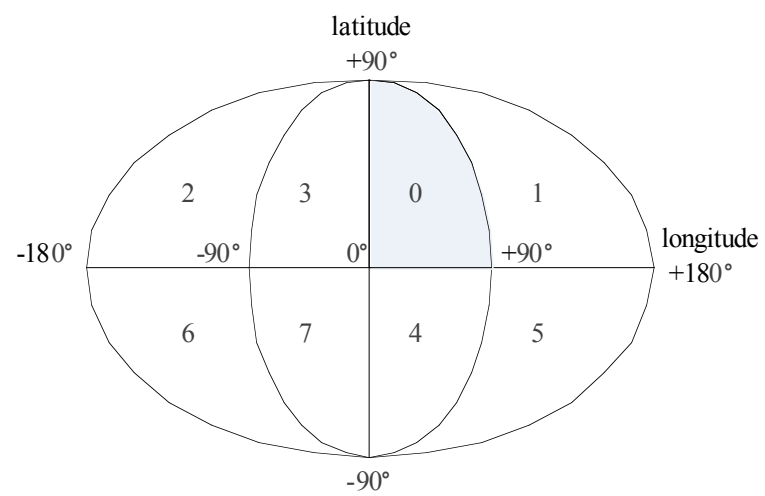

Fig. (10). Encoding rules of spherical octant.

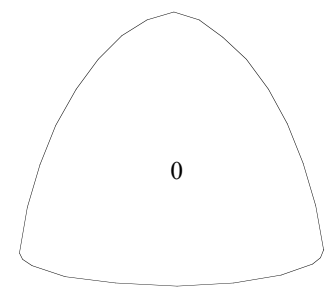

(a) Initial octant

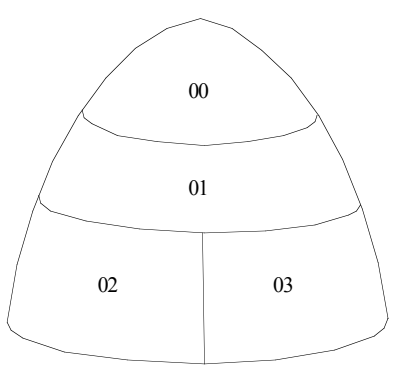

(b) First subdivision level

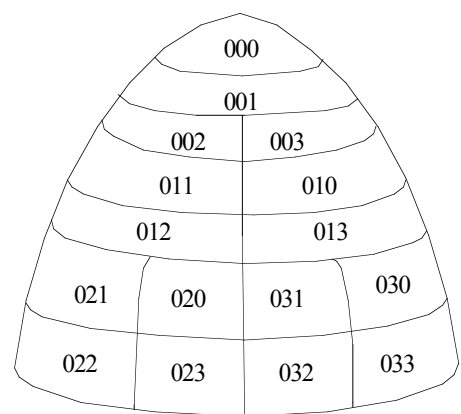

(c) Second subdivision level

Fig. (11). Morton encoding of grid cells.
The each figure of Morton code is a quaternary number which is not bigger than 3 . The number of the digits will increase by 1 after each subdivision. The more the subdivision levels are, the smaller the area of the sub-area is and the bigger the number of digits of corresponding Morton code is. The grid cell's Morton code can be expressed as:

$$
\begin{aligned}
& \text { Morton }=c_{1} c_{2} c_{3} \ldots c_{n}= \\
& c_{1} \cdot 10^{n}+c_{2} \cdot 10^{n-1}+c_{3} \cdot 10^{n-2}+\ldots+c_{n}
\end{aligned}
$$

\section{DISTORTION ANALYSIS OF SUBDIVISION GRID}

The geometric stability of grid, which is significant for maintaining the accuracy of the grid, is the basic characteristic of grid model. It directly affects the expression of geographic entities and operation accuracy under different resolutions. The geometric uniformity and stability under different resolution levels are important reference designators of selecting grid in practical applications.

The sides of the grid cells are the arcs of latitude and longitude lines, therefore, the arc length of each latitude line $L$ can be calculated by the following formula

\section{$L=2 \pi R \cos \varphi$}

Where $R$ is the radius of the Earth, $\varphi$ is the latitude of the arc. Take an equal-area quad-tree subdivision of the initial spherical triangle $R_{i}(\mathrm{i}=0,1,2 \ldots, 8)$ as an example, the initial arc length is:

$l=\frac{L}{8}$

For the $n t h$ level subdivision, the calculated arc length is:

$$
L_{n}=\frac{l}{4^{n}}
$$

The arc length $L_{m}$ of the longitude line between $\varphi_{1}$ and $\varphi_{2}$ can be calculated by the following formula:

$$
\begin{aligned}
& L_{m}=R\left(\varphi_{2}-\varphi_{1}\right)= \\
& R\left(\arcsin \left[\frac{F}{2 R^{2}\left(\lambda_{2}-\lambda_{1}\right)}+\sin \varphi_{1}\right]-\varphi_{1}\right)
\end{aligned}
$$

Where $\lambda_{1}$ and $\lambda_{2}$ are the longitude values of the subdivision grids.

The coordinates of grid nodes of each level are known, and the maximum side length $L_{n}$ and the minimum side length $L_{m}$ of each grid level can be obtained by solving the formula (3) and (4), thereby obtaining the distortion effect of the subdivision grid, and the calculation results are shown in Table 1.

As can be seen from Table 1, with the increase of subdivision levels, the ratio of the maximum and minimum side lengths of subdivision grid cells shows different trends, and the distortion of grid cells near the equator is large. However, with the increase of subdivision levels, the ratio converges to 2.0 (Fig. 12). The ratio of the maximum and 
Table 1. Changes of Side Lengths of Grid Cells in Different Subdivision Levels

\begin{tabular}{|c|c|c|c|c|c|}
\hline $\begin{array}{l}\text { Subdivision } \\
\text { level }\end{array}$ & The number of grids & $\begin{array}{l}\text { The area of the grid cell } \\
\qquad\left(m^{2}\right)\end{array}$ & $\begin{array}{l}\text { The maximum side } \\
\text { length }(\mathrm{m})\end{array}$ & $\begin{array}{l}\text { The minimum side } \\
\text { length }(\mathrm{m})\end{array}$ & $L_{n} / L_{m}$ \\
\hline 1 & 4 & 15975246396110.7000 & 6626783.141388 & 4338247.838017 & 1.527525 \\
\hline 2 & 16 & 3993811599027.6900 & 3486379.333861 & 2425154.388610 & 1.437591 \\
\hline 3 & 64 & 998452899756.9220 & 1764151.338501 & 1242522.135069 & 1.419815 \\
\hline 4 & 256 & 249613224939.2300 & 884677.838107 & 624948.620877 & 1.415601 \\
\hline 5 & 1024 & 62403306234.8076 & 442666.312314 & 312934.519000 & 1.414565 \\
\hline 6 & 4096 & 15600826558.7019 & 221379.499677 & 156526.655574 & 1.414325 \\
\hline 7 & 16384 & 3900206639.6755 & 110706.417341 & 78274.595774 & 1.414334 \\
\hline 8 & 65536 & 975051659.9189 & 55377.036404 & 39146.395518 & 1.414614 \\
\hline 9 & 262144 & 243762914.9797 & 27734.937595 & 19589.704889 & 1.415791 \\
\hline 10 & 1048576 & 60940728.7449 & 13959.774628 & 9827.588406 & 1.420468 \\
\hline 11 & 4194304 & 15235182.1862 & 7161.507396 & 4978.702293 & 1.438429 \\
\hline 12 & 16777216 & 3808795.5466 & 3923.027180 & 2615.139811 & 1.500121 \\
\hline 13 & 67108864 & 952198.8866 & 2532.958934 & 1533.566222 & 1.651679 \\
\hline 14 & 268435456 & 238049.7217 & 2042.625113 & 1109.073024 & 1.841741 \\
\hline 15 & 1073741824 & 59512.4304 & 1900.375654 & 974.473045 & 1.950157 \\
\hline 16 & 4294967296 & 14878.1076 & 1863.117219 & 937.809467 & 1.986669 \\
\hline$\ldots$ & $\ldots$ & $\ldots$ & $\ldots$ & $\ldots$ & $\ldots$ \\
\hline$>18$ & & & & & $<2.0$ \\
\hline
\end{tabular}

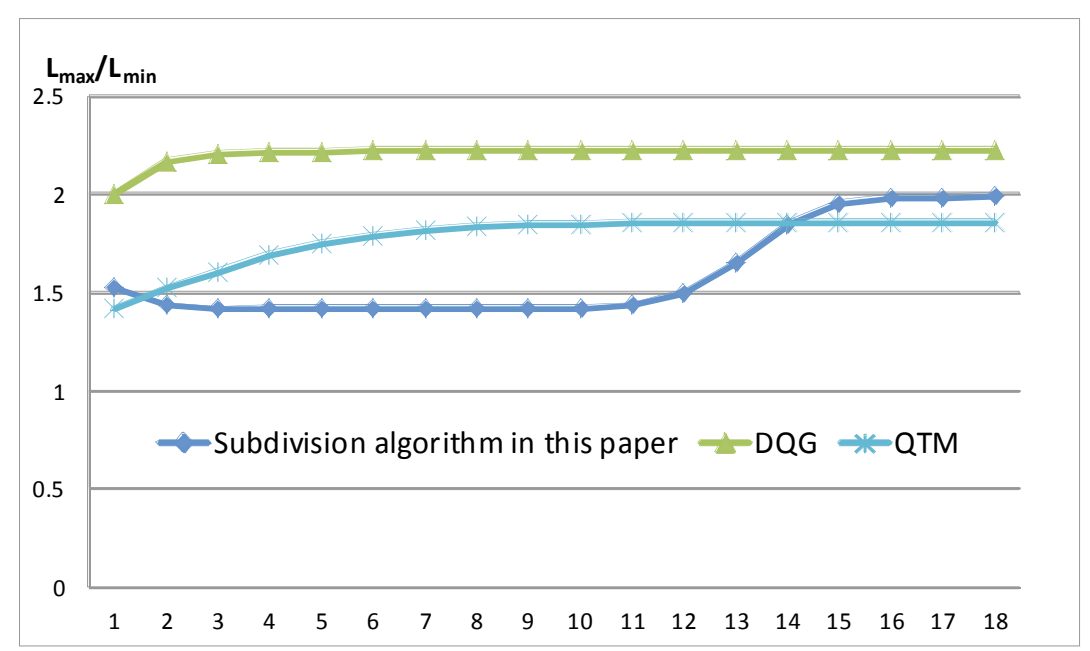

Fig. (12). Distortion ratio of different levels.

minimum edge of degenerate Quad-tree Grid (DQG) converges to 2.22 and does not exceed 2.23 [2]. Compared with DQG, the distortion property of the proposed grid subdivision improves slightly, and the convergence rate is better than DQG. Compared with Quaternary Triangular Mesh, although the grid cell distortion of the proposed method is a little bit higher than QTM (QTM converges to nearly 1.86 , not exceeding 1.9 [3]), it still has convergence - a feature that allows it to maintain quasi-uniform characteristics in its recursive subdivision.

Compared with the spherical QTM, DQG and latitude and longitude grid geometry, in addition to the advantage of stable geometric distortion mentioned above, the equal area global discrete grid subdivision method based on latitude and longitude lines also has the following characteristics: 1) The 
structure is simple. Its geometry structure is similar to a rectangular grid (except top triangles) and is simpler than that of the triangular grid, diamond-shaped grid and hexagonal grid. 2) Radial Symmetry and Translation Congruence. Except the top triangles, other quadrilateral cells will approximate rectangular grid with the increase of subdivision levels. The rectangular grid has radial symmetry and translation congruence, while the triangular grid does not have this feature. 3) Data Transferability. The corresponding data of rectangular cells can be converted to triangle, diamond and hexagonal grid cells by using statistical forecasting model.

\section{CONCLUSIONS}

In order to solve the shortcomings of the global discrete grid model, a novel equal area global discrete grid subdivision model based on latitude and longitude lines is proposed in this paper. The detailed subdivision process of the model is described and coding rules of grid cells are demonstrated. And the geometric distortion characteristics of grids with different subdivision hierarchy are also analyzed. Compared with other grid models, the proposed model has the following characteristics:

(1) The subdivision cells of each level (except that the 8 subdivision cells at two poles are spherical triangles) have the same geometrical shape, i.e. spherical quadrilateral. The structure is simple and the topological relationships are preserved. The unified worldwide positioning can be achieved and the requirements of global seamless grid hierarchy modelling can be met in a better way;

(2) The grid is compact and point distribution is more uniform. Meanwhile, the area of each cell of same subdivision level is equal, facilitating statistical calculations.

(3) The geometric distortion is stable. With the continuous refinement of the grid, the ratio of maximum and minimum side lengths of the grid cell converges to 2.0, maintaining an approximatelyuniform feature in the recursive subdivision. Its distortion threshold provides the basis for accuracy analysis and data quality control.

(4) The method has many properties which are conducive to hierarchical indexes, proximity search, coordinate conversion, data organization with multiresolution, compression storage, etc., such as consistent directionality, radial symmetry, translation congruence and data transferability.

In the digital spatial grid representation model of spherical surface, the proximity characteristic of data element is one of the most useful aspects of the GIS data structure, while proximity search is the foundation of spherical entity expansion and buffer analysis. The goal of our further research is to figure out an effective equal area quad-tree global discrete grid proximity search algorithm.

\section{CONFLICT OF INTEREST}

The authors confirm that this article content has no conflict of interest.

\section{ACKNOWLEDGEMENTS}

This work is supported by National Nature Science Foundation of China (No.61171051), Science and Technology Innovation Team Foundation of Zhengzhou city (No. 131PCXTD595).

\section{REFERENCES}

[1] HAO Xuesheng, WANG Lei,WANG Hongbin and LI Ying, "Modeling Methods and basic Problems of discrete Global grids," Geography and Geo-information Science, vol.28, no.1, pp.29-34, 2012.

[2] CUI Ma jun and ZHAO Xue sheng, "Tessellation and Distortion Analysis Based on Spherical DQG," Geography and GeoInformation Science,vol23, no.6, pp.23-25,2007.

[3] ZHAO Xuesheng, SUN Wenbin and CHEN Jun, "Distortion Distribution and Convergent Analysis of the Global Discrete Grid Based on QTM," Journal of China University of Mining\&Technology, vol34, no.4, pp.439-442, 2005.

[4] BAI Jianjun,WANG Lei,BAI Jiangtao,QIAO Yukang and QIAO Changlu, "An Equal Area Grid Model of WGS-84 Ellipsoidal Facet Based on EQG," Bulletin of Surveying and Mapping, vol.11, pp.1517, 2012.

[5] KOLAR J, "Representation of Geographic Terrain Surface Using Global Indexing," Proceedings of 12th International Conference on Geoinformatics, Sweden: Bridging the Pacific and Atllantic University of Gavle, pp.321-328, 2004.

[6] OU JiaMing, DU AiMin, E. THEB AULT, XU WenYao, TIAN XiaoBo and ZHANG TieLong, "A high resolution lithospheric magnetic field model over China," SCIENCE CHINA: Earth Sciences, vol.10, pp.1759-1768, 2013.

[7] H. Medellin, J. Corney and J.B.C. Davies, "Algorithms for the physical rendering and assembly of octree models," ComputerAided Design, vol.38, pp. 69-85, 2009.

[8] Cao, GF, Kyriakidis, PC and Goodchild, MF, "A multinomial logistic mixed model for the prediction of categorical spatial data," International Journal of Geographical Information Science, vol.25, no.12, pp. 2071-2086, 2011. 